\title{
Child, maternal and household-level correlates of nutritional status: a cross-sectional study among young Samoan children
}

\author{
Courtney C Choy ${ }^{1}$, Mayur M Desai ${ }^{1}$, Jennifer J Park ${ }^{1}$, Elizabeth A Frame ${ }^{2}$, \\ Avery A Thompson ${ }^{1}$, Take Naseri ${ }^{3}$, Muagututia S Reupena ${ }^{4}$, Rachel L Duckham ${ }^{5}$, \\ Nicole C Deziel ${ }^{1}$ and Nicola L Hawley ${ }^{1, *}$ \\ 'Department of Chronic Disease Epidemiology, Yale School of Public Health, Yale University, 60 College Street, \\ New Haven, CT 06520-8034, USA: ${ }^{2}$ University of Michigan School of Public Health, Ann Arbor, MI, USA: \\ ${ }^{3}$ Ministry of Health, Samoa: ${ }^{4}$ Samoa Bureau of Statistics, Samoa: ${ }^{5}$ Institute for Physical Activity and Nutrition \\ Research (IPAN), Deakin University, Melbourne, Australia
}

Submitted 11 August 2016: Final revision received 2 November 2016: Accepted 5 December 2016: First published online 6 February 2017

\begin{abstract}
Objective: Young children are particularly vulnerable to malnutrition as nutrition transition progresses. The present study aimed to document the prevalence, coexistence and correlates of nutritional status (stunting, overweight/obesity and anaemia) in Samoan children aged 24-59 months.

Design: A cross-sectional community-based survey. Height and weight were used to determine prevalence of stunting (height-for-age $Z$-score $<-2$ ) and overweight/ obesity (BMI-for-age $Z$-score $>+2$ ) based on WHO growth standards. Anaemia was determined using an AimStrip Hemoglobin test system $(\mathrm{Hb}<110 \mathrm{~g} / \mathrm{l})$.

Setting: Ten villages on the Samoan island of Upolu.

Subjects: Mother-child pairs ( $n$ 305) recruited using convenience sampling.

Results: Moderate or severe stunting was apparent in $20.3 \%$ of children, $16 \cdot 1 \%$ were overweight/obese and $34.1 \%$ were anaemic. Among the overweight/obese children, $28.6 \%$ were also stunted and $42.9 \%$ anaemic, indicating dual burden of malnutrition. Stunting was significantly less likely among girls (OR $=0 \cdot 41 ; 95 \%$ CI $0 \cdot 21,0.79, P<0.01)$ than boys. Overweight/obesity was associated with higher family socio-economic status and decreased sugar intake (OR per $10 \mathrm{~g} / \mathrm{d}=0.89$, $95 \%$ CI $0.80,0.99, P=0.032)$. The odds of anaemia decreased with age and anaemia was more likely in children with an anaemic mother $(\mathrm{OR}=2 \cdot 20 ; 95 \%$ CI $1.22,3.98, P=0.007)$. No child, maternal or household characteristic was associated with more than one of the nutritional status outcomes, highlighting the need for condition-specific interventions in this age group.

Conclusions: The observed prevalences of stunting, overweight/obesity and anaemia suggest that it is critical to invest in nutrition and develop health programmes targeting early childhood growth and development in Samoa.
\end{abstract}

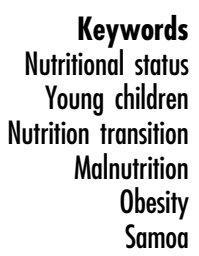

The nutrition transition, underlined by economic development, modernization and rapid urbanization ${ }^{(1)}$, has perpetuated the disproportionately high burden of noncommunicable diseases (NCD) in low- and middle-income countries $^{(2)}$. Patterns of diet and physical activity have shifted towards increased consumption of high-energy, nutrient-poor foods and increased sedentary activity, resulting in chronic energy imbalance and poor nutritional outcomes $^{(3)}$. The adverse health effects associated with this nutrition transition are often greatest in children under 5 years of age ${ }^{(4)}$. Changes in lifestyle often threaten appropriate development and growth in the earliest life stages, contributing to a higher risk of malnutrition in all its forms (under- and overnutrition) ${ }^{(5)}$ and premature NCD mortality ${ }^{(6)}$. Particularly in young children, malnutrition has been closely linked with increased mortality ${ }^{(7)}$ and impaired cognitive, physical and metabolic development ${ }^{(8)}$.

Samoa is a low- and middle-income country in the South Pacific undergoing significant nutrition transition, with shifts in societal and dietary structures along with significant rises in NCD prevalence over the past two decades $^{(9-11)}$. High levels of obesity and other associated metabolic disorders have been well documented among the Samoan adult population ${ }^{(12-14)}$. A high prevalence of overweight and obesity has also been observed among school-aged children. Data from 2003 showed $14.8 \%$ of boys and $6.3 \%$ of girls aged $6-8$ years to be overweight, while $3.3 \%$ of boys and $14.3 \%$ of girls were 
already obese ${ }^{(10)}$. While the burden of NCD is clear among adults and school-aged children, there remains a paucity of data about nutritional status in children under 5 years of age.

Present knowledge of child nutritional status in this age group relies on data from the Samoa National Nutrition Survey conducted over 15 years ago $(1999)^{(15)}$. Data from that survey showed that $4.2 \%$ of children aged 6-59 months were stunted and 1.9\% were underweight. At the same time, $10 \cdot 6 \%$ of children aged $24-59$ months were overweight or obese and $23.2 \%$ were anaemic $^{(15)}$. Since dramatic rises in obesity and concurrent problems with malnutrition have been identified in Samoan adults and school-aged children over the past 20 years ${ }^{(10,12,13,16)}$, a reassessment was warranted in young children.

The present study served to document the current nutritional status of Samoan children between 24 and 59 months old and to identify child, maternal and household-level correlates of malnutrition. The specific study aims were to: (i) to describe the prevalence and coexistence of four indicators of malnutrition, namely stunting, overweight/ obesity, anaemia and underweight; (ii) compare the prevalence of these nutritional indicators in three census regions with varying levels of urbanization and exposure to the nutrition transition; and (iii) examine associations between selected child, maternal and household characteristics and child nutritional status. Understanding the correlates of malnutrition is critical to informing future NCD control and prevention strategies targeted at this age group.

\section{Methods}

\section{Study population}

The recruitment and measurement of study participants took place from June to August 2015 on the island of Upolu, Samoa. The majority (76\%) of the Samoan population resides on Upolu, with the rest of the population residing on the more rural island of Savai'i and other smaller, outlying islands. From 285 villages within Upolu, ten villages were included in the study: four from the Apia Urban Area (AUA; an urban region), three from Northwest Upolu (NWU; a peri-urban region) and three from Rest of Upolu (ROU; a rural region). These villages were selected based on their prior participation in an adult obesity study $^{(12)}$. Exposure to the nutrition transition closely follows the gradient of urbanization across these regions, with AUA the most exposed and ROU the least. For the ease of data collection, we focused on large villages (population $>500$ ), paying close attention to geographic distribution across each region. The Ministry of Health and the Samoan Bureau of Statistics arranged for village-level permission to spend 2 to $3 \mathrm{~d}$ in each village and complete study activities. Recruitment was undertaken with the cooperation of village leaders and women's committee representatives, whose role was to explain the purpose and procedures of the study to women residing in the village and promote interest in participation. In each village, any mother and child pair that wanted to participate in the study came to a centrally located building in the village on the designated survey day. A field team of two investigators from Yale University, one investigator from the University of Michigan and two locally trained field assistants conducted the data collection.

A convenience sample of 319 mother and child pairs participated in the study. Since few data were available on prevalence of the health conditions being measured, the study was powered to detect an $8-10 \%$ difference in the prevalence of obesity compared with the 1999 Samoa National Nutrition Survey (estimate based on an increase in obesity of over $20 \%$ among adults over the same period). Three hundred dyads were needed to detect this difference at $80 \%$ power, $\alpha=0 \cdot 10$. Interest in participation exceeded that target, resulting in a sample size of 319 dyads.

Eligible children were 24-59 months old and of Samoan origin (based on having four Samoan grandparents). Eligible mothers were not pregnant (assessed by selfreport), had no severe physical or cognitive impairment (assessed by interviewers), and were willing and able to complete the interview portion of the study. Where mothers had two or more children who met study criteria, we randomly selected one for participation. For the present study, we excluded fourteen children: data checking identified two children under 24 months old, ten lacked complete physical measurements, and two were excluded based on having been born at <37 weeks' gestation to avoid the sequelae of prematurity biasing analyses of growth data. Therefore, the final sample for analyses included 305 mother and child pairs.

\section{Physical measurements}

Anthropometric measurements were taken from both mothers and children, with all participants in light island clothing. Standing height was measured to the nearest $0 \cdot 1 \mathrm{~cm}$ using a portable stadiometer (Pfister Imports, NY, USA) and weight to the nearest $0 \cdot 1 \mathrm{~kg}$ using a Tanita HD 351 digital weight scale (Tanita Corporation of America, IL, USA). Duplicate measures were averaged for use in analyses. A finger-prick blood $\mathrm{Hb}$ sample was also taken using an AimStrip Hb test system (Germaine Laboratories Inc., TX, USA).

\section{Child nutritional status outcomes}

The 2006 WHO growth standards were used to construct dichotomous indicators of stunting, overweight/obesity and underweight. Children whose height-for-age was below -2 SD, BMI-for-age was above +2 SD or weight-forage was below -2 SD from the corresponding median of the reference population (height-for-age $Z$-score (HAZ) $<-2$, BMI-for-age $Z$-score (BMIZ) $>+2$ or weight-for-age $Z$-score (WAZ) <-2) were classified as stunted, 
overweight/obese or underweight, respectively ${ }^{(17)}$. Child anaemia was defined as having $\mathrm{Hb}$ level below $110 \mathrm{~g} / \mathrm{l}^{(18)}$.

\section{Child, maternal and household characteristics}

A questionnaire, administered in Samoan to all mothers, collected detailed information on child, maternal and household characteristics.

Child characteristics included health history, infant feeding practices (breast-feeding and medication use), nutrition and maternal-reported physical activity. We used a dietary screener to assess general dietary behaviours and frequency, including eating breakfast daily and consuming specific types of foods, such as eating frozen desserts on a weekly basis. Dietary intake was assessed using a 104-item FFQ with a $30 \mathrm{~d}$ reference period. The questionnaire was based on a previously validated version of the instrument used in adults and school-aged children in Samoa and American Samoa ${ }^{(14)}$ and updated to include four child-specific foods (canned infant foods, puréed fruit/ vegetable pouches, infant formula and breast milk). The frequency of intake of individual items was measured across seven categories ranging from never/less than once per month to more than six times per day. We calculated daily total energy intake (TEI) and nutrients by multiplying the daily consumption frequency of each food by the nutrient content of a fixed, standard portion size. The nutrient content of FFQ items was estimated based on the US Department of Agriculture Food Composition Tables $^{(19)}$ and complementary information from the FAO Pacific Island Food Composition Tables ${ }^{(20)}$ for locally produced items. Macronutrient energy was divided by total energy to estimate the percentage of total energy from each macronutrient. Intakes of specific nutrients were adjusted for total energy using the residual method ${ }^{(21)}$.

We used the Netherlands Physical Activity Questionnaire for Young Children (NPAQ) ${ }^{(22)}$ to assess children's physical activity level. No locally relevant physical activity questionnaire has been developed for use in the Samoan population; therefore we used a questionnaire designed for use in another developed country setting in the same age group that had been widely used in other settings ${ }^{(22,23)}$. Mothers were asked to describe their child's level of physical activity and preferences for being active compared with other children using a series of seven 5-point Likert scale questions. Answers were summed to calculate a physical activity score, with a higher score indicating higher levels of activity. The physical activity score was categorized into approximate tertiles for analysis: 0-27 ( $n$ 109), 28-29 ( $n$ 84) and 30-35 ( $n$ 112). In addition, we calculated total screen time by summing the reported minutes spent watching television and using a computer or phone.

We collected data on maternal demographic characteristics, including age, highest level of education attained and marital status. Maternal weight status was classified as either normal $\left(<26 \mathrm{~kg} / \mathrm{m}^{2}\right)$ or overweight/obese $\left(\geq 26 \mathrm{~kg} / \mathrm{m}^{2}\right)$ based on Polynesian BMI cut-offs, which are sensitive to the greater lean mass per kilogram in the adult Polynesian population compared with those of other ethnicities ${ }^{(24)}$. Maternal anaemia was defined as $\mathrm{Hb}$ level less than $120 \mathrm{~g} / \mathrm{l}^{(18)}$.

For household characteristics, mothers were asked to select from five category ranges of annual household income, based on the 2011 Samoan Census ${ }^{(25)}$. Based on the distribution of responses, we dichotomized income into less than $\$ 10000$ tala $v$. $\$ 10000$ tala or more for the purpose of our analyses. A material lifestyle score (MLS) was calculated for each household based on ownership of eighteen consumer durables (such as television or refrigerator). This type of index is a sensitive measure of family socio-economic status in modernizing societies and has been previously used to study the adult Samoan population $^{(14)}$. We categorized the MLS into approximate quartiles based on the score distribution: 0-2 ( $n$ 63), 3-4 ( $n$ 68), 5-7 ( $n$ 97) and 8-18 ( $n$ 77). These quartiles are referred to as low, medium, high and very high MLS, respectively. Mothers' perception of the community spirit in their village of residence was measured using a 5-point Likert scale question from an adult Samoan study ${ }^{(12)}$, and categorized as weak, average or strong.

\section{Statistical methods}

The analysis for the study proceeded in the following steps. First, we used descriptive statistics to summarize the characteristics of the sample and then performed bivariate analyses to examine sample differences across the regions using the ANOVA $F$ test for continuous variables, the $\chi^{2}$ test for categorical variables and TEI-adjusted linear regression models for macronutrient and micronutrient intakes.

Second, we examined the unadjusted associations between the sample characteristics and each of the child nutritional status outcomes using $\chi^{2}$ tests, $t$ tests or TEIadjusted models, as appropriate.

Finally, multivariable logistic regression analyses were performed to identify independent child, maternal and household correlates of the child nutritional status outcomes. For each outcome, we used a backward elimination strategy to determine the most parsimonious model. Variables significant at the $0 \cdot 10$ level were retained in the final models, given the limited sample size. In addition, we included census region, child's age group and sex, and TEI in all models regardless of their statistical significance to ensure that observed associations were not confounded by these sample characteristics. All analyses were performed using the statistical software package SAS version 9.4.

\section{Results}

\section{Characteristics of the study population}

Mean age of the children and mothers was $40 \cdot 0$ (SD 10.2) months and $33 \cdot 8$ (sD 9.6) years, respectively. The number 
Table 1 Description of the study population by census region; Samoan island of Upolu, June-August 2015

\begin{tabular}{|c|c|c|c|c|c|c|c|c|c|}
\hline \multirow[b]{2}{*}{ Characteristic } & \multicolumn{2}{|l|}{$\begin{array}{l}\text { Total } \\
(n 305)\end{array}$} & \multicolumn{2}{|c|}{$\begin{array}{l}\text { Apia Urban Area } \\
\qquad(n 100)\end{array}$} & \multicolumn{2}{|c|}{$\begin{array}{l}\text { Northwest Upolu } \\
(n \text { 102) }\end{array}$} & \multicolumn{2}{|c|}{$\begin{array}{l}\text { Rest of Upolu } \\
\quad(n \text { 103) }\end{array}$} & \multirow[b]{2}{*}{$P+$} \\
\hline & $\%^{\star}$ or mean & SD & $\%^{*}$ or mean & SD & $\%^{*}$ or mean & SD & $\%^{*}$ or mean & SD & \\
\hline \multicolumn{10}{|l|}{ Child } \\
\hline Age (months) & & & & & & & & & 0.941 \\
\hline $24-35$ & $40 \cdot 0$ & & $43 \cdot 0$ & & 38.2 & & 38.8 & & \\
\hline $36-47$ & 33.8 & & 33.0 & & 33.3 & & $35 \cdot 0$ & & \\
\hline $48-59$ & $26 \cdot 2$ & & $24 \cdot 0$ & & 28.4 & & $26 \cdot 2$ & & \\
\hline Female & 48.5 & & 53.0 & & $49 \cdot 0$ & & 43.7 & & 0.412 \\
\hline First child & $24 \cdot 6$ & & $23 \cdot 0$ & & $26 \cdot 5$ & & $24 \cdot 3$ & & 0.845 \\
\hline Birth weight $(\mathrm{kg}) \ddagger$ & 3.6 & 0.5 & 3.6 & 0.6 & 3.6 & 0.5 & 3.6 & 0.6 & 0.868 \\
\hline Ever breast-fed & 68.2 & & $71 \cdot 0$ & & $72 \cdot 6$ & & $61 \cdot 2$ & & 0.165 \\
\hline Illness in the past 3 weeks & $9 . \overline{5}$ & & $12 \cdot 0$ & & $10 \cdot 8$ & & $5 \cdot \overline{8}$ & & 0.281 \\
\hline Deworming medication use in the past 3 weeks & 19.5 & & $25 \cdot 3$ & & $17 \cdot 8$ & & $15 \cdot 7$ & & 0.201 \\
\hline Ate breakfast daily in the past week & $66 \cdot 2$ & & $64 \cdot 0$ & & 65.7 & & 68.9 & & 0.751 \\
\hline Fruit consumption (times/week) & 7.3 & $6 \cdot 6$ & $6 \cdot 9$ & 5.5 & $6 \cdot 0$ & 4.9 & 9.0 & 8.4 & 0.003 \\
\hline Vegetable consumption (times/week) & 7.5 & 5.7 & $6 \cdot 4$ & $5 \cdot 2$ & $7 \cdot 0$ & 5.4 & $9 \cdot 2$ & $6 \cdot 1$ & 0.001 \\
\hline Sweetened fruit juice consumption (times/week) & 0.2 & 0.7 & 0.3 & $0 . \overline{8}$ & 0.3 & 0.8 & $0 . \overline{1}$ & 0.4 & 0.150 \\
\hline Frozen dessert consumption (times/week) & 1.8 & 1.8 & $2 \cdot 1$ & $2 \cdot 2$ & 1.7 & 1.5 & 1.6 & 1.4 & 0.059 \\
\hline Night-time sleep $(\mathrm{h} / \mathrm{d})$ & 9.5 & 1.0 & $9 \cdot 7$ & 1.4 & 9.5 & 0.9 & $9 \cdot 3$ & 0.7 & 0.034 \\
\hline Total screen time $(\mathrm{min} / \mathrm{d}) \ddagger$ & 79.9 & 55.9 & 90.5 & $60 \cdot 0$ & 61.5 & $47 \cdot 7$ & 87.9 & $55 \cdot 2$ & $<0.001$ \\
\hline Physical activity score (NPAQ)§ & & & & & & & & & 0.014 \\
\hline Tertile $1($ mean $=24 \cdot 1, \mathrm{SD}=3 \cdot 8)$ & 35.7 & & $34 \cdot 0$ & & 43.1 & & $30 \cdot 1$ & & \\
\hline Tertile $2($ mean $=29.1, \mathrm{SD}=0.7)$ & 27.5 & & $26 \cdot 0$ & & $33 \cdot 3$ & & $23 \cdot 3$ & & \\
\hline Tertile $3($ mean $=32 \cdot 0, \mathrm{SD}=1.2)$ & $36 \cdot 7$ & & $40 \cdot 0$ & & 23.5 & & $46 \cdot 6$ & & \\
\hline \multicolumn{10}{|l|}{ Mother } \\
\hline Age (years) & 33.9 & $9 \cdot 6$ & 34.9 & $10 \cdot 3$ & 34.6 & $9 \cdot 4$ & $32 \cdot 0$ & 8.9 & 0.054 \\
\hline High-school graduate & $60 \cdot 3$ & & $70 \cdot 0$ & & $62 \cdot 8$ & & 48.5 & & 0.007 \\
\hline Married or cohabitating & $76 \cdot 1$ & & $72 \cdot 0$ & & $76 \cdot 5$ & & 79.6 & & 0.443 \\
\hline Overweight/obese $\left(\mathrm{BMl}>26 \mathrm{~kg} / \mathrm{m}^{2}\right)$ & $87 \cdot 2$ & & $90 \cdot 0$ & & $85 \cdot 3$ & & 86.4 & & 0.579 \\
\hline Anaemia $(\mathrm{Hb}>120 \mathrm{~g} / \mathrm{l})$ & $22 \cdot 7$ & & $19 \cdot 0$ & & $15 \cdot 7$ & & 33.3 & & 0.006 \\
\hline \multicolumn{10}{|l|}{ Household } \\
\hline Annual income less than $\$ 10000$ tala & 78.3 & & $66 \cdot 7$ & & 83.3 & & 84.5 & & 0.003 \\
\hline Material lifestyle score $\|$ & & & & & & & & & $<0.001$ \\
\hline Quartile $1($ mean $=1.3, \mathrm{SD}=0.8)$ & $20 \cdot 7$ & & $15 \cdot 0$ & & 24.5 & & $22 \cdot 3$ & & \\
\hline Quartile $2($ mean $=3.5, \mathrm{SD}=0.5)$ & $22 \cdot 3$ & & $17 \cdot 0$ & & $22 \cdot 6$ & & $27 \cdot 2$ & & \\
\hline Quartile $3($ mean $=5.9, \mathrm{sD}=0.8)$ & 31.8 & & $26 \cdot 0$ & & 33.3 & & $35 \cdot 9$ & & \\
\hline Quartile $4($ mean $=10.5, \mathrm{SD}=2.8)$ & $25 \cdot 3$ & & $42 \cdot 0$ & & $19 \cdot 6$ & & 14.6 & & \\
\hline Community spirit & & & & & & & & & 0.033 \\
\hline Weak & $2 \cdot 3$ & & 3.0 & & 0.0 & & 3.9 & & \\
\hline Average & 48.4 & & 49.5 & & $56 \cdot 9$ & & 38.8 & & \\
\hline Strong & $49 \cdot 3$ & & 47.5 & & 43.1 & & 57.3 & & \\
\hline
\end{tabular}

*Percentages may not sum to $100 \%$ due to missing data.

$\dagger P$ values for ANOVA $F$ test (for continuous variables) or $x^{2}$ test (for categorical variables).

†Total screen time is the sum of the reported minutes spent watching television, on the computer or on the phone.

§Physical activity score is the sum of items from the Netherlands Physical Activity Questionnaire for Young Children (NPAQ). The score is out of a maximum of 35 , with higher scores indicating greater physical activity.

$\|$ Material lifestyle score is a sum of consumer durables owned (fridge, freezer, stereo, portable stereo, microwave oven, rice cooker, blender, sewing machine, television, VCR/DVD, couch, washing machine, landline telephone, computer/laptop, tablet, electric fan, air conditioner and motor vehicle). The score is out of a maximum of 18 , with higher scores indicating higher material lifestyle.

of mother-child pairs was similar across the urban AUA ( $n$ 100), peri-urban NWU ( $n$ 102) and rural ROU ( $n$ 103) regions.

Table 1 presents the characteristics of the children and mothers by census region. The distribution of child age and sex did not differ between census regions. Based on data from the dietary screener, we found significant differences in children's fruit and vegetable consumption across regions $(P<0.01$ for both), with the highest levels in the rural ROU region and lowest in the urban AUA region. Rural ROU children were also more active, based on their NPAQ scores, than children in the other regions. Urban AUA children had the highest mean total screen time $(90.5$ (SD 60.0) $\mathrm{min} / \mathrm{d})$. Level of maternal education varied across census regions, with the most high-school graduates in the urban AUA (70.0\%) and the least in the rural ROU region (48.5\%). Urban AUA households were wealthier and had a higher MLS compared with those in the peri-urban NWU and rural ROU regions.

Child daily dietary intake, based on the FFQ, is presented in Table 2. Mean daily TEI was significantly different across the three census regions, with the children living in the rural ROU region having the lowest total energy intake $(10924 \mathrm{~kJ} / \mathrm{d}$ $(2611.0 \mathrm{kcal} / \mathrm{d}) v .12409 \mathrm{~kJ} / \mathrm{d}(2965.8 \mathrm{kcal} / \mathrm{d})$ in AUA and $18960 \mathrm{~kJ} / \mathrm{d}$ ( $4531.6 \mathrm{kcal} / \mathrm{d})$ in NWU; Table 2), although the energy intake reported in all regions far exceeded the caloric recommendations for this age group ${ }^{(26)}$. Sugar intake also differed by census region, with the NWU 
Table 2 Daily energy, macronutrient and micronutrient intakes of child participants by census region*; Samoan island of Upolu, June-August 2015

\begin{tabular}{|c|c|c|c|c|c|c|c|c|c|}
\hline & \multicolumn{2}{|c|}{$\begin{array}{c}\text { Total } \\
(n \text { 305) }\end{array}$} & \multicolumn{2}{|c|}{$\begin{array}{l}\text { Apia Urban Area } \\
(n 100)\end{array}$} & \multicolumn{2}{|c|}{$\begin{array}{l}\text { Northwest Upolu } \\
(n \text { 102) }\end{array}$} & \multicolumn{2}{|c|}{$\begin{array}{l}\text { Rest of Upolu } \\
\qquad(n 103)\end{array}$} & \multirow[b]{2}{*}{$P+$} \\
\hline & Mean & SD & Mean & SD & Mean & SD & Mean & SD & \\
\hline TEI (kJ) & $14098 \cdot 4$ & $10937 \cdot 0$ & $12408 \cdot 9$ & $8101 \cdot 9$ & $18960 \cdot 2$ & $14493 \cdot 0$ & $10924 \cdot 4$ & $6942 \cdot 9$ & $<0.001$ \\
\hline \multicolumn{10}{|l|}{ Intake as a percentage of TEI } \\
\hline Total protein & 14.4 & $3 \cdot 6$ & 14.4 & $5 \cdot 7$ & $13 \cdot 2$ & 5.9 & $15 \cdot 7$ & 5.7 & $<0.001$ \\
\hline Total carbohydrates & $52 \cdot 8$ & $6 \cdot 2$ & $52 \cdot 5$ & $10 \cdot 5$ & $55 \cdot 0$ & $10 \cdot 7$ & $51 \cdot 0$ & $10 \cdot 4$ & $<0.001$ \\
\hline Total fat & $35 \cdot 3$ & $4 \cdot 0$ & $35 \cdot 5$ & 6.9 & $34 \cdot 7$ & $7 \cdot 1$ & $35 \cdot 5$ & 6.9 & 0.259 \\
\hline \multicolumn{10}{|l|}{ TEI-adjusted nutrient intake } \\
\hline Saturated fat $(\mathrm{g})$ & $46 \cdot 0$ & $8 \cdot 6$ & $45 \cdot 2$ & $7 \cdot 7$ & $46 \cdot 2$ & 8.4 & $46 \cdot 5$ & 9.4 & 0.546 \\
\hline Monounsaturated fat $(\mathrm{g})$ & 29.2 & 6.5 & $30 \cdot 1$ & $6 \cdot 7$ & 28.9 & $6 \cdot 2$ & $28 \cdot 6$ & 6.5 & 0.193 \\
\hline Polyunsaturated fat $(\mathrm{g})$ & $16 \cdot 5$ & $3 \cdot 6$ & $16 \cdot 9$ & $3 \cdot 2$ & $15 \cdot 8$ & 3.6 & $16 \cdot 9$ & 4.0 & 0.052 \\
\hline Fibre $(\mathrm{g})$ & $36 \cdot 2$ & $7 \cdot 6$ & $35 \cdot 1$ & 7.6 & 37.6 & 6.9 & $36 \cdot 0$ & $8 \cdot 1$ & 0.067 \\
\hline Sugar (g) & $145 \cdot 6$ & $36 \cdot 0$ & $142 \cdot 0$ & $31 \cdot 8$ & 159.6 & 33.7 & $135 \cdot 3$ & 38.0 & $<0.001$ \\
\hline Cholesterol (mg) & $364 \cdot 6$ & 123.9 & 357.5 & $125 \cdot 7$ & $342 \cdot 8$ & $112 \cdot 8$ & 393.2 & $128 \cdot 2$ & 0.011 \\
\hline $\mathrm{Na}(\mathrm{mg})$ & 3141.7 & $631 \cdot 2$ & $3167 \cdot 7$ & $597 \cdot 2$ & $3020 \cdot 6$ & 597.5 & 3236.5 & 680.6 & 0.043 \\
\hline Vitamin A (IU) & $2618 \cdot 3$ & $1758 \cdot 1$ & $2432 \cdot 2$ & $1590 \cdot 5$ & $2864 \cdot 3$ & $1842 \cdot 0$ & 2555.4 & $1816 \cdot 3$ & 0.197 \\
\hline Vitamin A, retinol $(\mathrm{mg})$ & 785.5 & $527 \cdot 4$ & $729 \cdot 7$ & $477 \cdot 2$ & $859 \cdot 3$ & $552 \cdot 6$ & 766.6 & 544.9 & 0.197 \\
\hline Vitamin $A, \beta$-carotene $(\mathrm{mg})$ & $1571 \cdot 0$ & 1054.9 & $1459 \cdot 3$ & $954 \cdot 3$ & $1718 \cdot 6$ & $1105 \cdot 2$ & $1533 \cdot 2$ & $1089 \cdot 8$ & 0.197 \\
\hline Vitamin E (mg) & 11.7 & $2 \cdot 7$ & 11.4 & 2.5 & $11 \cdot 6$ & $2 \cdot 8$ & $12 \cdot 0$ & $2 \cdot 8$ & 0.243 \\
\hline Vitamin C (mg) & 247.4 & $87 \cdot 1$ & $232 \cdot 6$ & $78 \cdot 0$ & 276.4 & 90.5 & 233.1 & $85 \cdot 6$ & $<0.001$ \\
\hline $\mathrm{Fe}(\mathrm{mg})$ & $16 \cdot 6$ & $2 \cdot 3$ & $16 \cdot 9$ & $2 \cdot 4$ & $16 \cdot 7$ & $2 \cdot 0$ & $16 \cdot 2$ & 2.5 & 0.131 \\
\hline $\mathrm{Ca}(\mathrm{mg})$ & 755.7 & 194.9 & 743.0 & $177 \cdot 2$ & 814.0 & 163.4 & $710 \cdot 4$ & $225 \cdot 0$ & $<0.001$ \\
\hline $\mathrm{K}(\mathrm{mg})$ & $4602 \cdot 8$ & $811 \cdot 2$ & 4503.4 & 824.7 & $4623 \cdot 7$ & $752 \cdot 6$ & $4678 \cdot 6$ & $851 \cdot 1$ & 0.292 \\
\hline
\end{tabular}

TEl, total energy intake.

*All nutrient intakes adjusted for TEI based on child FFQ data.

$\dagger P$ values for ANOVA $F$ test (for TEI and amount of nutrient intake per day) or TEl-adjusted linear regression (for macronutrient intake as a percentage of TEl).

region consuming the most $(159.6 \mathrm{~g} / \mathrm{d})$ and the most rural ROU region consuming the least $(135 \cdot 3 \mathrm{~g} / \mathrm{d})$. Among the micronutrients, only TEI-adjusted mean daily intakes of vitamin $\mathrm{C}, \mathrm{Ca}$, cholesterol and $\mathrm{Na}$ differed across regions; daily intakes of vitamin $\mathrm{C}$ and $\mathrm{Ca}$ were greatest among peri-urban NWU children while mean daily cholesterol and $\mathrm{Na}$ intakes were greatest among rural ROU children.

\section{Associations between child, maternal and bousebold characteristics and child nutritional status}

The overall prevalence of stunting, overweight/obesity and anaemia was $20.3 \%$ ( $n$ 62), 16.1\% ( $n$ 49) and 34.1\% ( $n$ 104), respectively. Only seven children were underweight ( $2.3 \%$; data not shown) and therefore characteristics associated with underweight are not described in the current paper. Despite the differences in child, maternal and household characteristics described above, census region was not significantly associated with any nutritional status outcome. Tables 3 and 4 show the unadjusted associations between sample characteristics and nutritional status outcomes. Any characteristic with an unadjusted association with the outcomes at a significance level of 0.01 was carried forward into the multivariable models reported in Table 5.

Odds of child stunting were approximately 59\% lower for females compared with males, after adjusting for other characteristics $(P=0 \cdot 006)$. Children who ate frozen desserts at least weekly had $70 \%$ lower odds of stunting compared with those who did not $(P<0 \cdot 001)$. For every $10 \mathrm{mg}$ increase in daily vitamin $C$ consumption, we found $4 \%$ increased odds of stunting $(P=0 \cdot 046)$. While eating breakfast daily was associated with stunting in the bivariate analysis, the association was not significant in the multivariable model $(\mathrm{OR}=2.09$; $95 \%$ CI 0.93, 4.68, $P=0.073)$.

Children with very high MLS had nearly fourfold greater odds of overweight/obesity compared with those with low MLS (OR $=3 \cdot 61 ; 95 \%$ CI 1.22, 10.71, $P=0 \cdot 016)$. The odds of overweight/obesity decreased by $11 \%$ for every $10 \mathrm{~g}$ increase in daily sugar intake $(P=0.032)$.

After adjusting for other characteristics, odds of child anaemia decreased with age. Among children aged 48-59 months, the odds were $75 \%$ lower than among children who were $24-35$ months old $(P<0 \cdot 001)$. For every $100 \mathrm{mg}$ increase in daily $\mathrm{Ca}$ intake, the odds of anaemia increased by $14 \%(P=0.052)$. Children with a married mother had a lower odds of anaemia (OR $=0.60 ; 95 \% \mathrm{CI}$ $0.34,1.07)$ than children with single mothers, although the association was not significant $(P=0.084)$. Children with an anaemic mother had more than twice the odds of anaemia compared with children whose mother's Hb level was within the healthy range $(\mathrm{OR}=2 \cdot 20 ; 95 \% \mathrm{CI} 1 \cdot 22,3 \cdot 98, P=0.007)$.

\section{Dual burden of malnutrition}

Although levels of stunting and anaemia did not differ significantly between children who were overweight/obese and those who were not $(P=0 \cdot 118$ and $P=0 \cdot 175$, respectively), among the forty-nine overweight and obese children, 
Table 3 Unadjusted associations between sample characteristics and child nutritional status outcomes; Samoan island of Upolu, JuneAugust 2015

\begin{tabular}{|c|c|c|c|c|}
\hline Characteristic & $n^{*}$ & $\begin{array}{c}\text { Stunting } \\
(H A Z<-2) \\
(\%)\end{array}$ & $\begin{array}{c}\mathrm{OW} / \mathrm{OB} \\
(\mathrm{BMIZ}>+2) \\
(\%)\end{array}$ & $\begin{array}{c}\text { Anaemia } \\
(\mathrm{Hb}<110 \mathrm{~g} / \mathrm{l}) \\
(\%)\end{array}$ \\
\hline Total & 305 & $20 \cdot 3$ & $16 \cdot 1$ & $34 \cdot 1$ \\
\hline \multicolumn{5}{|l|}{ Census region } \\
\hline Apia Urban Area & 100 & $18 \cdot 0$ & $22 \cdot 0$ & $35 \cdot 0$ \\
\hline Northwest Upolu & 102 & $17 \cdot 7$ & $11 \cdot 8$ & 31.4 \\
\hline Rest of Upolu & 103 & $25 \cdot 2$ & $14 \cdot 6$ & $35 \cdot 9$ \\
\hline$P \dagger$ & & 0.313 & 0.124 & 0.769 \\
\hline \multicolumn{5}{|l|}{ Child } \\
\hline \multicolumn{5}{|l|}{ Age (months) } \\
\hline $24-35$ & 122 & 33.6 & 18.9 & $42 \cdot 6$ \\
\hline $36-47$ & 103 & $20 \cdot 4$ & $16 \cdot 5$ & $38 \cdot 8$ \\
\hline $48-59$ & 80 & 0.0 & $11 \cdot 3$ & $15 \cdot 0$ \\
\hline$P+$ & & $<0.001$ & 0.351 & $<0.001$ \\
\hline Female & 148 & $15 \cdot 5$ & $15 \cdot 5$ & $35 \cdot 8$ \\
\hline Male & 157 & $24 \cdot 8$ & $16 \cdot 6$ & $32 \cdot 5$ \\
\hline$P \dagger$ & & 0.044 & 0.808 & 0.540 \\
\hline First child & 75 & $21 \cdot 3$ & $13 \cdot 3$ & 34.7 \\
\hline Not & 230 & $20 \cdot 0$ & $17 \cdot 0$ & 33.9 \\
\hline$P+$ & & 0.803 & 0.458 & 0.905 \\
\hline \multicolumn{5}{|l|}{ Reported birth weight } \\
\hline $2500 \mathrm{~g}$ or less (low) & 7 & $14 \cdot 3$ & 0.0 & $28 \cdot 6$ \\
\hline 2501-3999 g (normal) & 206 & 21.4 & $15 \cdot 1$ & 32.5 \\
\hline $4000 \mathrm{~g}$ or more (macrosomia) & 69 & $15 \cdot 9$ & $20 \cdot 3$ & $33 \cdot 3$ \\
\hline$P+$ & & 0.579 & 0.298 & 0.963 \\
\hline Ever breast-fed & 208 & $17 \cdot 3$ & $15 \cdot 4$ & $33 \cdot 2$ \\
\hline Not & 97 & $26 \cdot 8$ & $17 \cdot 5$ & $36 \cdot 1$ \\
\hline$P \dagger$ & & 0.055 & 0.635 & 0.618 \\
\hline Illness in the past 3 weeks & 29 & $17 \cdot 2$ & $17 \cdot 2$ & $49 \cdot 3$ \\
\hline No & 276 & 20.7 & $15 \cdot 9$ & $32 \cdot 6$ \\
\hline$P \dagger$ & & 0.664 & 0.856 & 0.090 \\
\hline Deworming medication use in the past 3 weeks & 59 & 23.7 & $20 \cdot 3$ & $44 \cdot 1$ \\
\hline No & 243 & $19 \cdot 8$ & $15 \cdot 2$ & 31.7 \\
\hline$P \dagger$ & & 0.498 & 0.339 & 0.072 \\
\hline Ate breakfast daily in the past week & 202 & $24 \cdot 8$ & $17 \cdot 8$ & $37 \cdot 1$ \\
\hline No & 103 & 11.7 & $12 \cdot 6$ & $28 \cdot 2$ \\
\hline$P \dagger$ & & 0.007 & 0.242 & 0.118 \\
\hline Eats fruits daily & 109 & $26 \cdot 6$ & $18 \cdot 4$ & $37 \cdot 6$ \\
\hline No & 196 & $16 \cdot 8$ & $14 \cdot 8$ & $32 \cdot 1$ \\
\hline$P+$ & & 0.042 & 0.418 & 0.334 \\
\hline Eats vegetables daily & 138 & $25 \cdot 4$ & $17 \cdot 4$ & 37.0 \\
\hline No & 167 & $16 \cdot 2$ & $15 \cdot 0$ & 31.7 \\
\hline$P \dagger$ & & 0.047 & 0.567 & 0.339 \\
\hline Drinks sweetened fruit juice daily & 42 & $7 \cdot 1$ & $19 \cdot 1$ & $38 \cdot 1$ \\
\hline No & 263 & $22 \cdot 4$ & $15 \cdot 6$ & 33.5 \\
\hline$P+$ & & 0.022 & 0.571 & 0.556 \\
\hline Eats frozen desserts weekly & 234 & $15 \cdot 0$ & $15 \cdot 0$ & $31 \cdot 2$ \\
\hline No & 71 & $38 \cdot 0$ & $19 \cdot 7$ & $43 \cdot 7$ \\
\hline$P+$ & & $<0.001$ & 0.339 & 0.052 \\
\hline \multicolumn{5}{|l|}{ Night-time sleep $(\mathrm{h} / \mathrm{d})$} \\
\hline 8 or less & 29 & $17 \cdot 2$ & 6.9 & $20 \cdot 7$ \\
\hline $9-10$ & 223 & $20 \cdot 2$ & $17 \cdot 9$ & 35.4 \\
\hline More than 10 & 47 & 23.4 & 14.9 & $36 \cdot 2$ \\
\hline$P \dagger$ & & 0.800 & 0.305 & 0.275 \\
\hline \multicolumn{5}{|l|}{ Total screen time $(\mathrm{min} / \mathrm{d})$} \\
\hline Less than 60 & 54 & $24 \cdot 1$ & $13 \cdot 0$ & 31.5 \\
\hline $60-119$ & 171 & $19 \cdot 9$ & 17.5 & 34.5 \\
\hline $120-179$ & 58 & $20 \cdot 7$ & $10 \cdot 3$ & 34.5 \\
\hline 180 or more & 22 & 13.6 & $27 \cdot 3$ & $36 \cdot 4$ \\
\hline $\mathrm{P} \dagger$ & & 0.777 & 0.249 & 0.972 \\
\hline \multicolumn{5}{|l|}{ Physical activity score } \\
\hline Tertile $1($ mean $=24 \cdot 1, \mathrm{SD}=3 \cdot 8)$ & 109 & 23.9 & $18 \cdot 4$ & $31 \cdot 2$ \\
\hline Tertile $2($ mean $=29.1, \mathrm{sD}=0.7)$ & 84 & $19 \cdot 1$ & $13 \cdot 1$ & $39 \cdot 3$ \\
\hline Tertile $3($ mean $=32 \cdot 0, \mathrm{SD}=1 \cdot 2)$ & 112 & $17 \cdot 9$ & $16 \cdot 1$ & 33.0 \\
\hline$P+$ & & 0.511 & 0.615 & 0.478 \\
\hline
\end{tabular}




\begin{tabular}{|c|c|c|c|c|}
\hline Characteristic & $n^{*}$ & $\begin{array}{c}\text { Stunting } \\
(\mathrm{HAZ}<-2) \\
(\%)\end{array}$ & $\begin{array}{c}\text { OW/OB } \\
(\mathrm{BMIZ}>+2) \\
(\%)\end{array}$ & $\begin{array}{c}\text { Anaemia } \\
(\mathrm{Hb}<110 \mathrm{~g} / \mathrm{l}) \\
(\%)\end{array}$ \\
\hline \multicolumn{5}{|l|}{ Mother } \\
\hline \multicolumn{5}{|l|}{ Age (years) } \\
\hline $18-24.99$ & 68 & $26 \cdot 5$ & 14.7 & $42 \cdot 7$ \\
\hline $25-39.99$ & 155 & 21.3 & $14 \cdot 2$ & 34.8 \\
\hline $40+$ & 82 & $13 \cdot 4$ & $20 \cdot 7$ & $25 \cdot 6$ \\
\hline$P+$ & & 0.129 & 0.403 & 0.087 \\
\hline High-school graduate & 184 & $16 \cdot 3$ & 18.5 & $32 \cdot 1$ \\
\hline Not & 121 & $26 \cdot 5$ & 12.4 & $37 \cdot 2$ \\
\hline $\mathrm{P}+$ & & 0.031 & 0.157 & 0.356 \\
\hline Married or cohabitating & 232 & 19.4 & $16 \cdot 0$ & 31.0 \\
\hline Not & 73 & 23.3 & $16 \cdot 4$ & 43.8 \\
\hline$P+$ & & 0.471 & 0.921 & 0.044 \\
\hline OW/OB $\left(\mathrm{BMI} \geq 26.0 \mathrm{~kg} / \mathrm{m}^{2}\right)$ & 266 & $18 \cdot 8$ & $16 \cdot 9$ & 33.5 \\
\hline No & 39 & $30 \cdot 8$ & $10 \cdot 3$ & 38.5 \\
\hline Pt & & 0.083 & 0.290 & 0.538 \\
\hline Any anaemia & 69 & 27.5 & 18.8 & $49 \cdot 3$ \\
\hline No & 235 & $18 \cdot 3$ & $15 \cdot 3$ & $29 \cdot 4$ \\
\hline$P+$ & & 0.094 & 0.484 & 0.002 \\
\hline \multicolumn{5}{|l|}{ Household } \\
\hline Annual income less than $\$ 10000$ tala & 238 & 23.1 & $16 \cdot 4$ & $35 \cdot 3$ \\
\hline$\$ 10000$ tala or more & 66 & $10 \cdot 6$ & $15 \cdot 2$ & $30 \cdot 3$ \\
\hline Pf & & 0.026 & 0.809 & 0.450 \\
\hline \multicolumn{5}{|l|}{ Material lifestyle score } \\
\hline Quartile $1($ mean $=1.3, \mathrm{SD}=0.8)$ & 63 & $19 \cdot 1$ & 7.9 & 34.9 \\
\hline Quartile 2 (mean $=3.5, \mathrm{sD}=0.5$ ) & 68 & $29 \cdot 4$ & 14.7 & $35 \cdot 3$ \\
\hline Quartile $3($ mean $=5.9, \mathrm{sD}=0.8)$ & 97 & $18 \cdot 6$ & 14.4 & $32 \cdot 0$ \\
\hline Quartile 4 (mean $=10.5, \mathrm{sD}=2 \cdot 8$ ) & 77 & $15 \cdot 6$ & $26 \cdot 0$ & $35 \cdot 1$ \\
\hline $\mathrm{Pt}$ & & 0.188 & 0.030 & 0.962 \\
\hline \multicolumn{5}{|l|}{ Community spirit } \\
\hline Weak & 7 & 42.9 & $14 \cdot 3$ & 28.6 \\
\hline Average & 147 & $21 \cdot 8$ & $17 \cdot 0$ & $36 \cdot 1$ \\
\hline Strong & 150 & 18.0 & $15 \cdot 3$ & 32.7 \\
\hline$P+$ & & 0.237 & 0.918 & 0.785 \\
\hline
\end{tabular}

$\mathrm{HAZ}$, height-for-age Z-score; OW/OB, overweight and obesity; BMIZ, BMI-for-age $Z$ score.

${ }^{*}$ Numbers may not sum to totals due to missing data.

$\dagger P$ value for $x^{2}$ test.

$28.6 \%$ ( $n$ 14) were also classified as stunted and $42.9 \%$ ( $n$ 21) were anaemic (data not shown). Of the sixty-two children who were stunted, 48.4\% ( $n$ 30) were anaemic; however, this proportion of anaemia was significantly lower than in those who were not stunted $(51.6 \%, n 32 ; P=0.008)$.

\section{Discussion}

Adequate nutritional status is critical for child growth and development and health later in life. The present study identified a worrisome prevalence of malnutrition among young Samoan children, which needs to be addressed with targeted intervention. While child, maternal and household characteristics differed by census region, there were no differences in the prevalence of stunting, overweight/obesity or anaemia by census region, indicating that, despite differential exposure to urbanization and the nutrition transition, all Samoan children are at risk of poor nutritional outcomes. Several child, maternal and household characteristics were identified to be associated with each nutritional status outcome, giving suggestions for intervention targets.
The prevalence of stunting (20.3\%), overweight/obesity (16.1\%) and anaemia (34.1\%) observed in the current study was substantially higher than reported in the 1999 Samoan National Nutrition Survey. While the two studies are not directly comparable, as the National Nutrition Survey included children living on the island of Savai'i, which is the most rural area of Samoa, the increased prevalence of malnutrition reported in the current study suggests that the development, modernization and nutrition transition that have taken place in Samoa over the past 16 years has not benefited child nutritional status. This is in line with other settings in which urbanization and nutritional transition have had detrimental effects on child health $^{(2)}$ because it exposes children to diets of higher energy content, but lower quality ${ }^{(4)}$ and an increased burden of infectious disease associated with lower income and more crowded, urban housing ${ }^{(27)}$.

Stunting reflects chronic undernutrition during the most critical periods of growth and development in early life. Overall, the prevalence of stunting observed in the present study was consistent with reports from other Pacific Island nations: $48 \%$ of children under 5 years of age are 
Table 4 Child daily dietary intake by nutritional status outcomes $(n 305)^{\star}$; Samoan island of Upolu, June-August 2015

\begin{tabular}{|c|c|c|c|c|c|c|c|c|c|c|c|c|c|c|c|}
\hline & \multicolumn{2}{|c|}{$\begin{array}{c}\text { Stunting } \\
(\mathrm{HAZ}<-2) \\
(n 62)\end{array}$} & \multicolumn{2}{|c|}{$\begin{array}{l}\text { No stunting } \\
\text { (HAZ } \geq-2) \\
(\text { (n 243) }\end{array}$} & \multirow[b]{2}{*}{$P+$} & \multicolumn{2}{|c|}{$\begin{array}{c}\mathrm{OW} / \mathrm{OB} \\
(\mathrm{BMIZ}>+2) \\
(n 49)\end{array}$} & \multicolumn{2}{|c|}{$\begin{array}{l}\text { No OW/OB } \\
(\mathrm{BMI} \leq+2) \\
(n 256)\end{array}$} & \multirow[b]{2}{*}{$P \dagger$} & \multicolumn{2}{|c|}{$\begin{array}{c}\text { Any anaemia } \\
(\mathrm{Hb}<110 \mathrm{~g} / \mathrm{l}) \\
(n 104)\end{array}$} & \multicolumn{2}{|c|}{$\begin{array}{c}\text { No anaemia } \\
(\mathrm{Hb} \geq 110 \mathrm{~g} / \mathrm{l}) \\
(\text { n 201) }\end{array}$} & \multirow[b]{2}{*}{$P \dagger$} \\
\hline & Mean & SD & Mean & SD & & Mean & SD & Mean & SD & & Mean & SD & Mean & SD & \\
\hline $\begin{array}{l}\text { TEI (kJ) } \\
\text { Intake as a percentage of TEI }\end{array}$ & 14211.4 & $9986 \cdot 8$ & $14069 \cdot 5$ & 11185.5 & 0.293 & $13984 \cdot 2$ & $9806 \cdot 5$ & $14120 \cdot 2$ & $11157 \cdot 9$ & 0.931 & $14283 \cdot 3$ & $11161 \cdot 2$ & $14002 \cdot 6$ & $10846 \cdot 2$ & 0.832 \\
\hline $\begin{array}{l}\text { Intake as a percentage of IEl } \\
\text { Total protein }\end{array}$ & 14.9 & 7.5 & $14 \cdot 3$ & 3.8 & 0.210 & 14.7 & 8.5 & 14.4 & 3.7 & 0.540 & $14 \cdot 2$ & 5.8 & 14.6 & 4.2 & 0.411 \\
\hline Total carbohydrates & $52 \cdot 3$ & $13 \cdot 6$ & 52.9 & 6.9 & 0.446 & 51.5 & $15 \cdot 3$ & 53.0 & $6 \cdot 7$ & 0.108 & $53 \cdot 2$ & $10 \cdot 5$ & $52 \cdot 6$ & $7 \cdot 6$ & 0.382 \\
\hline Total fat & $35 \cdot 2$ & $8 \cdot 8$ & $35 \cdot 3$ & 4.5 & 0.851 & $36 \cdot 1$ & 9.9 & $35 \cdot 0$ & $4 \cdot 3$ & 0.100 & $35 \cdot 1$ & $6 \cdot 8$ & 35.4 & 4.9 & 0.522 \\
\hline \multicolumn{16}{|l|}{ TEI-adjusted nutrient intake } \\
\hline Saturated fat $(\mathrm{g})$ & $46 \cdot 5$ & $10 \cdot 6$ & $45 \cdot 8$ & $8 \cdot 0$ & 0.639 & $48 \cdot 0$ & 8.7 & $45 \cdot 6$ & 8.5 & 0.066 & $46 \cdot 3$ & $9 \cdot 2$ & $45 \cdot 8$ & $8 \cdot 2$ & 0.577 \\
\hline Monounsaturated fat (g) & $28 \cdot 8$ & $7 \cdot 6$ & $29 \cdot 3$ & $6 \cdot 1$ & 0.627 & $29 \cdot 8$ & $6 \cdot 2$ & $29 \cdot 1$ & 6.5 & 0.439 & $28 \cdot 4$ & $7 \cdot 0$ & $29 \cdot 6$ & $6 \cdot 1$ & 0.118 \\
\hline Polyunsaturated fat $(\mathrm{g})$ & $15 \cdot 7$ & 4.3 & $16 \cdot 7$ & $3 \cdot 4$ & 0.102 & $17 \cdot 0$ & 3.9 & $16 \cdot 4$ & 3.6 & 0.256 & $16 \cdot 1$ & 3.8 & $16 \cdot 7$ & 3.5 & 0.156 \\
\hline Fibre $(\mathrm{g})$ & 36.9 & $7 \cdot 6$ & $36 \cdot 1$ & $7 \cdot 6$ & 0.458 & 35.4 & $6 \cdot 7$ & 36.4 & $7 \cdot 7$ & 0.411 & $36 \cdot 2$ & $7 \cdot 6$ & $36 \cdot 3$ & $7 \cdot 6$ & 0.942 \\
\hline Sugar $(g)$ & 139.9 & 39.3 & $147 \cdot 1$ & $35 \cdot 1$ & 0.157 & $135 \cdot 2$ & $31 \cdot 1$ & 147.6 & $36 \cdot 6$ & 0.026 & $145 \cdot 8$ & 35.6 & $145 \cdot 5$ & 36.4 & 0.944 \\
\hline Cholesterol (mg) & 368.8 & 133.3 & 363.6 & 121.6 & 0.768 & 391.7 & $137 \cdot 2$ & 359.4 & $120 \cdot 7$ & 0.095 & $362 \cdot 1$ & $127 \cdot 4$ & 365.9 & $122 \cdot 3$ & 0.801 \\
\hline $\mathrm{Na}(\mathrm{mg})$ & 3193.7 & $807 \cdot 0$ & $3128 \cdot 4$ & $579 \cdot 1$ & 0.552 & $3255 \cdot 2$ & $660 \cdot 6$ & $3120 \cdot 0$ & 624.4 & 0.170 & 3093.0 & 653.8 & $3166 \cdot 9$ & $619 \cdot 3$ & 0.333 \\
\hline Vitamin A (IU) & 3069.9 & $2260 \cdot 1$ & 2503.0 & $1590 \cdot 5$ & 0.067 & 2734.5 & 1659.5 & $2596 \cdot 0$ & $1778 \cdot 6$ & 0.614 & 2578.0 & $1947 \cdot 0$ & $2639 \cdot 1$ & 2578.0 & 0.774 \\
\hline Vitamin A, retinol (mg) & 921.0 & 678.0 & 750.9 & $477 \cdot 2$ & 0.067 & $820 \cdot 4$ & 497.9 & 778.8 & 533.6 & 0.614 & 773.4 & 584.1 & 791.7 & 497.0 & 0.774 \\
\hline Vitamin $A, \beta$-carotene (mg) & 1841.9 & $1356 \cdot 1$ & $1501 \cdot 8$ & 954.3 & 0.067 & $1640 \cdot 7$ & 995.7 & $1557 \cdot 6$ & $1067 \cdot 2$ & 0.614 & $1546 \cdot 8$ & $1168 \cdot 2$ & 1583.5 & 994.0 & 0.774 \\
\hline Vitamin E (mg) & 11.6 & 2.7 & 11.7 & 2.7 & 0.847 & $11 \cdot 3$ & 2.5 & 11.7 & $2 \cdot 7$ & 0.366 & 11.4 & 2.9 & 11.8 & 2.6 & 0.200 \\
\hline Vitamin C (mg) & $270 \cdot 7$ & $113 \cdot 0$ & 241.5 & $78 \cdot 3$ & 0.058 & 238.2 & $92 \cdot 3$ & 249.2 & $86 \cdot 1$ & 0.412 & $250 \cdot 0$ & 88.7 & $246 \cdot 1$ & $86 \cdot 4$ & 0.709 \\
\hline $\mathrm{Fe}(\mathrm{mg})$ & $16 \cdot 6$ & $3 \cdot 1$ & $16 \cdot 6$ & $2 \cdot 1$ & 0.910 & $17 \cdot 3$ & 2.6 & 16.5 & $2 \cdot 3$ & 0.027 & $16 \cdot 9$ & $2 \cdot 8$ & $16 \cdot 4$ & $2 \cdot 0$ & 0.131 \\
\hline $\mathrm{Ca}(\mathrm{mg})$ & 790.5 & $276 \cdot 2$ & $746 \cdot 8$ & $167 \cdot 6$ & 0.238 & $802 \cdot 4$ & 194.7 & $746 \cdot 8$ & 194.0 & 0.067 & 784.9 & $236 \cdot 7$ & $740 \cdot 6$ & 167.9 & 0.091 \\
\hline $\mathrm{K}(\mathrm{mg})$ & $4807 \cdot 3$ & 796.9 & $4550 \cdot 6$ & $808 \cdot 2$ & 0.026 & 4590.0 & 696.3 & $4605 \cdot 2$ & 832.6 & 0.904 & 4615.4 & 898.1 & $4596 \cdot 3$ & 764.7 & 0.846 \\
\hline
\end{tabular}

HAZ, height-for-age Z-score; OW/OB, overweight and obesity; BMIZ, BMI-for-age $Z$ score; TEI, total energy intake.

*Values based on child FFQ data.

† $P$ value for $t$ test (for TEI and amount of nutrient intake per day) or TEI-adjusted linear regression (for macronutrient intake as percentage of TEI). 
Table 5 Multivariable logistic regression models of sample characteristics associated with child stunting, overweight/obesity and anaemia; Samoan island of Upolu, June-August 2015

\begin{tabular}{|c|c|c|c|c|c|c|c|c|c|}
\hline \multirow[b]{2}{*}{ Characteristic } & \multicolumn{3}{|c|}{ Stunting ( $n$ 305) } & \multicolumn{3}{|c|}{ OW/OB (n 305) } & \multicolumn{3}{|c|}{ Anaemia $(n 304)^{*}$} \\
\hline & $\begin{array}{l}\text { Adjusted } \\
\text { OR }\end{array}$ & $95 \% \mathrm{Cl}$ & $P$ & $\begin{array}{l}\text { Adjusted } \\
\text { OR }\end{array}$ & $95 \% \mathrm{Cl}$ & $P$ & $\begin{array}{l}\text { Adjusted } \\
\text { OR }\end{array}$ & $95 \% \mathrm{Cl}$ & $P$ \\
\hline \multicolumn{10}{|l|}{ Census region } \\
\hline Apia Urban Area & 1.00 & Ref. & - & 1.00 & Ref. & - & 1.00 & Ref. & - \\
\hline North West Upolu & 0.74 & $0.32,1.75$ & 0.497 & 0.62 & $0.26,1.47$ & 0.277 & 0.80 & $0.42,1.54$ & 0.500 \\
\hline Rest of Upolu & 1.22 & $0.55,2.71$ & 0.623 & 0.72 & $0.33,1.58$ & 0.414 & 1.04 & $0.56,1.95$ & 0.895 \\
\hline \multicolumn{10}{|l|}{ Child } \\
\hline \multicolumn{10}{|l|}{ Age (months) } \\
\hline 24-35 & 1.00 & Ref. & - & 1.00 & Ref. & - & 1.00 & Ref. & - \\
\hline $36-47$ & 0.56 & $0.28,1.11$ & 0.096 & 0.88 & $0.43,1.83$ & 0.739 & 0.88 & $0.50,1.54$ & 0.654 \\
\hline 48-59 & - & - & - & 0.65 & $0.27,1.56$ & 0.334 & 0.25 & $0.12,0.53$ & $<0.001$ \\
\hline \multicolumn{10}{|l|}{ Sex } \\
\hline Male & 1.00 & Ref. & _- & 1.00 & Ref. & _- & 1.00 & Ref. & - \\
\hline Female & 0.41 & $0.21,0.79$ & 0.006 & 0.89 & $0.47,1.70$ & 0.723 & $1 \cdot 18$ & $0.71,1.96$ & 0.531 \\
\hline TEl $(k J)$ & 1.00 & $1.00,1.00$ & 0.485 & 1.00 & $1.00,1.00$ & 0.643 & 1.00 & $1.00,1.00$ & 0.383 \\
\hline Eats frozen desserts weekly & 0.30 & $0.14,0.64$ & $<0.001$ & & & & & & \\
\hline Ate breakfast daily & 2.09 & $0.93,4.68$ & 0.073 & & & & & & \\
\hline Vitamin C (per $10 \mathrm{mg} / \mathrm{d})$ & 1.04 & $1.00,1.07$ & 0.046 & & & & & & \\
\hline $\mathrm{Ca}($ per $100 \mathrm{mg} / \mathrm{d})$ & & & & $1 \cdot 16$ & $1.00,1.34$ & 0.056 & 1.14 & $1.00,1.30$ & 0.052 \\
\hline Sugar (per $10 \mathrm{~g} / \mathrm{d})$ & & & & 0.89 & $0.80,0.99$ & 0.032 & & & \\
\hline \multicolumn{10}{|l|}{ Mother and household } \\
\hline \multicolumn{10}{|l|}{ Material lifestyle score } \\
\hline Quartile $1($ mean $=1.3, S D=0.8)$ & & & & 1.00 & Ref. & - & & & \\
\hline Quartile $2($ mean $=3.5, \mathrm{sD}=0.5)$ & & & & 1.75 & $0.54,5 \cdot 70$ & 0.824 & & & \\
\hline Quartile 3 (mean $=5.9, \mathrm{sD}=0.8$ ) & & & & 1.98 & $0.67,5.89$ & 0.856 & & & \\
\hline Quartile $4($ mean $=10 \cdot 5, \mathrm{sD}=2 \cdot 8)$ & & & & 3.61 & $1.22,10.71$ & 0.016 & & & \\
\hline Married or cohabitating & & & & & & & 0.60 & $0.34,1.07$ & 0.084 \\
\hline Maternal anaemia & & & & & & & $2 \cdot 20$ & $1.22,3.98$ & 0.007 \\
\hline
\end{tabular}

OW/OB, overweight and obesity; TEI, total energy intake; ref., reference category.

*One participant was excluded due to a missing value for maternal anaemia.

reportedly stunted in Papua New Guinea, 33\% in the Solomon Islands and $26.3 \%$ in Vanuatu ${ }^{(28)}$. The majority of the Samoan children who were classified as stunted were in the youngest age group of 24-35.99 months, while there was no stunting in the oldest age group. Although these data are cross-sectional, they suggest that Samoan children may regain position on the WHO height growth charts as they get older. This requires confirmation with longitudinal data, and further information on the potential consequences of this pattern of slower height growth for later health and cognitive development is also needed.

Young girls in the current study had decreased odds of stunting. This finding is consistent with studies in many other settings globally ${ }^{(29-31)}$ and across the Pacific, including Papua New Guinea ${ }^{(32)}$ and Vanuatu(28). Our data did not suggest that this was a result of different infant feeding practices or current nutritional status, and there is little evidence from the Pacific to suggest that daughters are preferred ${ }^{(33)}$, although this has not been exhaustively explored in contemporary populations. It has, however, been argued that improved growth in female compared with male children may reflect their relative resilience to adverse in utero environments ${ }^{(34)}$, seasonal fluctuations in nutritional availability and infectious diseases ${ }^{(32)}$.

Eating frozen desserts (such as ice cream) at least weekly was also found to be associated with decreased odds of child stunting; however, we suspect that this may reflect higher family socio-economic status and the child's overall energy intake, rather than specific nutritional content obtained from the desserts. In line with this hypothesis, consuming frozen desserts at least weekly was associated with higher MLS and mean daily TEI (data not shown). Contrary to the existing literature ${ }^{(35-37)}$, stunting was positively associated with the child eating breakfast daily (significant in the bivariate model and $P=0.07$ in the multivariable model) and daily intake of vitamin $C$. These findings are difficult to explain and warrant further investigation. There are inherent limitations of dietary intake estimation based on FFQ in children ${ }^{(38,39)}$, which may have contributed to these findings, particularly as we were not able to determine the dietary composition of breakfast specifically. Additionally, we were not able to account for reverse causality. Physicians may have already detected or parents may have recognized the low height-for-age among the stunted children in our sample and, in response, parents may have increased the child's dietary intake.

Looking at the opposite end of the spectrum of malnutrition, we found problematic levels of childhood overweight/obesity. This is especially concerning since overweight children are known to be at increased risk of becoming overweight adolescents and adults ${ }^{(40)}$ and of experiencing metabolic dysregulation in later life $^{(6)}$. 
Estimates of overweight/obesity among young children in Pacific Island nations are difficult to compare as different classifications for weight status, such as weight-for-height, weight-for-age or BMI-for-age ${ }^{(17)}$, have been used. However, in American Samoa, which is further along in the nutrition transition, it is estimated that approximately $35 \%$ of children under 5 years of age are overweight/obese using comparable WHO BMI-for-age $Z$-scores ${ }^{(41)}$. In the Commonwealth of the Northern Mariana Islands, childhood obesity was estimated to be approximately $25 \%$ in 2-3-year-olds and $26 \%$ in $4-6$-year-olds ${ }^{(42)}$. The strongest correlate of overweight/obesity in our sample was household MLS. High family socio-economic status, indicated by higher MLS score, increased the odds of overweight/obesity among young children, a pattern similar to that found among adults in this setting ${ }^{(10,13,14)}$.

High BMI-for-age often results from a chronic positive energy balance ${ }^{(5,17)}$; however, we did not find any differences in either maternal-reported physical activity level or mean daily TEI between young Samoan children who were overweight/obese and those who were not. Furthermore, there were no differences in the proportion of energy consumed from macronutrients (protein, carbohydrates and total fat) between the two groups. Interestingly, TEI-adjusted Ca intake was associated with increased odds of overweight/obesity in our bivariate analyses, consistent with the suggestion that dietary $\mathrm{Ca}$ intake may positively influence energy balance, particularly in overweight/obese children, although it did not reach the threshold of significance in our multivariable model ${ }^{(43)}$. Overall, dietary sugar intake was very high among children in our sample. In addition, we found an inverse association between sugar intake and overweight/obesity; however, this finding should be interpreted with caution, as we cannot differentiate between added $v$. natural sugars in the foods consumed by the child. Local fruits and vegetables, and starchy staples in the traditional Pacific Island diets, are very carbohydratedense $^{(20)}$, and these natural sugars contribute significantly to overall daily sugar intake. Commercial, processed food and beverage products may be nutritionally detrimental, potentially increasing dietary consumption of added sugar and displacing consumption of other more nutritious options in early years of life ${ }^{(13,44)}$. As may be the case with stunting, there could be issues of reverse causality if parents had recognized overweight and implemented dietary changes.

We found that more than one-third of the study sample (children and mothers) had some degree of anaemia. We included mild anaemia in our outcome, as the WHO cautions that even mild levels of anaemia may be problematic for growth and development in children under 5 years of age ${ }^{(18)}$. The prevalence of anaemia among the Samoan children was consistent with estimates from other countries across the Pacific region. In the Solomon Islands, $48 \%$ of children aged $6-59$ months are anaemic ${ }^{(28)}$, while more than half of children of the same age group are anaemic in Nauru ${ }^{(45)}$ and Tuvalu ${ }^{(28)}$. In neighbouring
American Samoa, the prevalence of anaemia is very similar in this age group: $33 \%$ among those aged $1-4$ years ${ }^{(46)}$. Also similar is the relationship between anaemia and age, with the odds of anaemia decreasing with age in both settings ${ }^{(46)}$. The odds of any anaemia were nearly $120 \%$ higher among children with an anaemic mother compared with those without, as expected based on the strong evidence linking maternal and child nutritional status ${ }^{(47)}$. Having a married mother was protective against child anaemia, and this supports the link between good health and nutrition behaviours in children with social support provided through marriage ${ }^{(48,49)}$. Increasing $\mathrm{Ca}$ intake increased the odds of child anaemia (although the association was just above $P<0.05(P=0.052)$ in the final model); this finding may reflect the competition between $\mathrm{Ca}$ and Fe for absorption in the body, which can adversely impact $\mathrm{Hb}$ levels, although, again, we acknowledge the limitations of the FFQ in measuring child dietary intake ${ }^{(50)}$.

While Fe deficiency is the most common nutritional source of anaemia in young children ${ }^{(51)}$, the current study did not find an association between the two, suggesting that perhaps the anaemia has an infectious cause. Multiple mosquito-borne illnesses and helminth infections, such as hookworms, can result in anaemia ${ }^{(52)}$ and further investigation is urgently needed to determine the source of anaemia among these young children. A recent study suggested that hookworms were present in the faeces of almost all dogs in Samoa $(97.5 \%)^{(53)}$; with a large and increasing population of stray dogs, this may represent a major public health concern.

The finding of coexisting levels of child stunting, overweight/obesity and anaemia highlights the vulnerability of young Samoan children to malnutrition in various forms, simultaneously. As in other low- and middle-income countries undergoing the nutrition transition, it is common to find a dual burden of malnutrition: undernourishment and micronutrient deficiencies, coexisting with the other extreme of overweight/obesity and other diet-related chronic diseases ${ }^{(54,55)}$. Obesity and micronutrient deficiencies are each problematic, but in combination exacerbate the severity of nutrition-related $\mathrm{NCD}^{(56)}$. Previous studies have consistently shown an association between childhood stunting and anaemia $^{(47,50,51)}$. Especially among the poor and those living in environments with insufficient sanitation, children are at higher risk of infection and micronutrient deficiencies ${ }^{(50)}$. While the global prevalence of undernutrition decreased between 1990 and 2012, the number of countries with equivalent levels of stunting and overweight among children under 5 years has increased substantially ${ }^{(57)}$.

Surprisingly, no common correlates of child nutritional status outcomes were observed in the present study. This finding suggests that different intervention approaches may be necessary to prevent malnutrition among young children in this setting. The study identified unique child, maternal and household characteristics associated with child nutritional status, which can potentially help us to 
identify those at risk of poor nutritional outcomes and target interventions ${ }^{(58)}$. Further study is needed to fully understand the origins of stunting and anaemia in this setting, including a comprehensive assessment of the possible infectious causes. However, overweight/obesity prevention efforts are clearly needed for this age group and can begin in the households at high risk.

The current study had some limitations. The findings may not be generalizable, as the study purposely recruited similar numbers of mother-child pairs from each of the three census regions and was therefore not nationally representative. The demographics, community layout and urban-rural setting on the Samoan island of Upolu may make any associations specific to this area in particular. As a result of our convenience sampling, there is the possibility that the prevalence estimates of stunting, overweight/obesity, underweight and/or anaemia may have been either under- or over-represented. Comparing our study sample with the 2014 Samoan Demographic and Health Survey showed that our participants were less educated than the general population $(60.3 \%$ had secondary education compared with $76 \%$ of the general population) and were of slightly lower socio-economic status based on household ownership of consumer durables ${ }^{(59)}$. Additionally, due to the cross-sectional approach of the study, we are unable infer causality and tease apart temporality to determine potential prospective risk factors for the child nutritional status outcomes; further longitudinal studies are needed to do this.

To our knowledge, the present study is the first regional study to report child, maternal and household characteristics associated with child nutritional status outcomes in children aged 2-5 years. Poor nutrition at this age has lifelong implications, reducing a child's ability to learn and grow to her/his full potential, and can lead to decreased productivity and weaker long-term national economic performance ${ }^{(5,58)}$. Nutrition-related NCD control and prevention strategies that target undernutrition and overweight/obesity simultaneously in this age group are urgently needed in Samoa and potentially elsewhere in the Pacific Islands. An investment in nutrition is foundational for human development and will help to ensure good health throughout the life course.

\section{Acknowledgements}

Acknowledgements: The authors would like to thank the study participants. They are grateful to the Samoan Ministry of Health (especially Christina Soti-Ulberg and staff at the Office of Nutrition Surveillance), Samoa Bureau of Statistics, and Ministry of Women, Community and Social Development for their support and facilitation of the study. Financial support: This work was supported by the David Dull Internship Fund (Yale University; CCC); the Stolwijk Fellowship (Yale University; J.J.P.); and Yale University and
University of Michigan Faculty Funds. The funders had no role in the design, analysis or writing of this article. Conflict of interest: None. Authorship: C.C.C. and N.L.H. conceived the study and, with M.M.D., wrote the initial draft of the manuscript. C.C.C., J.J.P. and E.A.F. conducted the research with the support of T.N., M.S.R., N.C.D. and R.L.D. C.C.C., M.M.D., A.A.T. and N.L.H. analysed and interpreted the data. All authors read and approved the final manuscript. Ethics of buman subject participation: This study was conducted according to the guidelines laid down in the Declaration of Helsinki and all procedures involving human subjects were approved by the Yale University Institutional Review Board and the Health Research Committee of the Samoan Ministry of Health. Written informed consent was obtained from all participants.

\section{References}

1. Popkin BM (1998) The nutrition transition and its health implications in lower-income countries. Public Health Nutr 1, 5-21.

2. Popkin BM (2006) Global nutrition dynamics: the world is shifting rapidly toward a diet linked with noncommunicable diseases. Am J Clin Nutr 84, 289-298.

3. Popkin BM (2004) The nutrition transition: an overview of world patterns of change. Nutr Rev 62, 7 Pt 2, S140-S143.

4. Drewnowski A \& Popkin BM (1997) The nutrition transition: new trends in the global diet. Nutr Rev 55, 31-43.

5. UNICEF, World Health Organization \& World Bank (2015) UNICEF-WHO-World Bank Group Joint Child Malnutrition Estimates. New York, Geneva and Washington, DC: UNICEF, WHO and World Bank.

6. Anderson I (2013) The Economic Costs of NonCommunicable Diseases in the Pacific Islands: A Rapid Stock Take of the Situation in Samoa, Tonga, and Vanuatu. Health, Nutrition, and Population (HNP) Discussion Paper. Washington, DC: World Bank Group.

7. Pelletier DL, Frongillo EA Jr, Schroeder DG et al. (1995) The effects of malnutrition on child mortality in developing countries. Bull World Health Organ 73, 443.

8. Walker SP, Wachs TD, Gardner JM et al. (2007) Child development: risk factors for adverse outcomes in developing countries. Lancet 369, 145-157.

9. Baker PT, Hanna JM \& Baker TS (editors) (1986) The Changing Samoans: Behavior and Health in Transition. New York: Oxford University Press.

10. Keighley ED, McGarvey ST, Quested C et al. (2007) Nutrition and health in modernizing Samoans: temporal trends and adaptive perspectives. In Health Change in the Asia-Pacific Region: Biocultural and Epidemiological Approaches, pp. 147-191 [R Ohtsuka and SJ Ulijaszek, editors]. New York: Cambridge University Press.

11. McGarvey ST (1991) Obesity in Samoans and a perspective on its etiology in Polynesians. Am J Clin Nutr 53, 6 Suppl., 1586S-1594S

12. Hawley NL, Minster RL, Weeks DE et al. (2014) Prevalence of adiposity and associated cardiometabolic risk factors in the Samoan genome-wide association study. Am J Hum Biol 26, 491-501.

13. Seiden A, Hawley NL, Schulz D et al. (2012) Long-term trends in food availability, food prices, and obesity in Samoa. Am J Hum Biol 24, 286-295.

14. DiBello JR, McGarvey ST, Kraft P et al. (2009) Dietary patterns are associated with metabolic syndrome in adult Samoans. J Nutr 139, 1933-1943. 
15. Mackerras D \& Kiernan DM (2003) Samoa National Nutritional Survey 1999. Part 3: Child Growth, Diet, Contact with the Health System and Interview with Carers. Technical Report. Apia, Samoa: Nutrition Centre.

16. NCD Risk Factor Collaboration (2016) Trends in adult bodymass index in 200 countries from 1975 to 2014: a pooled analysis of 1698 population-based measurement studies with 19.2 million participants. Lancet 387, 1377-1396.

17. de Onis M (2006) WHO Child Growth Standards: Length/ Height-for-Age, Weight-for-Age, Weight-for-Length, Weightfor-Height and Body Mass Index-for-Age. Geneva: WHO.

18. World Health Organization (2011) Haemoglobin Concentrations for the Diagnosis of Anaemia and Assessment of Severity. Vitamin and Mineral Nutrition Information System. Geneva: WHO.

19. US Department of Agriculture, Agricultural Research Service (2012) USDA National Nutrient Database for Standard Reference, Release 25. Nutrient Data Laboratory Home Page. http://www.ars.usda.gov/ba/bhnrc/ndl (accessed February 2016).

20. Dignan C, Burlingame B, Kumar S et al. (2004) The Pacific Islands Food Composition Tables. Rome: FAO.

21. Willett WC, Howe GR \& Kushi LH (1997) Adjustment for total energy intake in epidemiologic studies. Am J Clin Nutr 65, 4 Suppl., 1220S-1228S.

22. Janz KF, Broffitt B \& Levy SM (2005) Validation evidence for the Netherlands physical activity questionnaire for young children: the Iowa bone development study. Res $Q$ Exerc Sport 76, 363-369.

23. Bielemann RM, Reichert FF, Paniz VM et al. (2011) Validation of the Netherlands physical activity questionnaire in Brazilian children. Int J Behav Nutr Phys Act 8, 45.

24. Swinburn BA, Ley SJ, Carmichael HE et al. (1999) Body size and composition in Polynesians. Int J Obes Relat Metab Disord 23, 1178-1183.

25. Samoa Bureau of Statistics (2011) Population and Housing Census 2011: Analytical Report. Apia, Samoa: CensusSurveys and Demography Division, SBS, Government of Samoa.

26. Institute of Medicine (2005) Dietary Reference Intakes for Energy, Carbohydrate, Fiber, Fat, Fatty Acids, Cholesterol, Protein, and Amino Acids (Macronutrients). Washington, DC: The National Academy Press.

27. Alirol E, Getaz L, Stoll B et al. (2011) Urbanisation and infectious diseases in a globalised world. Lancet Infect Dis 11, 131-141.

28. Grieve H, Busch-Hallen J \& Mellor K (2013) Development of the Nutritional Critical Appraisal Tool (NCAT): Briefing Paper. Australia: Compass: Women's and Children's Health Knowledge Hub.

29. Wamani H, Astrom AN, Peterson S et al. (2007) Boys are more stunted than girls in sub-Saharan Africa: a metaanalysis of 16 demographic and health surveys. BMC Pediatr 7, 17.

30. Hien NN \& Kam S (2008) Nutritional status and the characteristics related to malnutrition in children under five years of age in Nghean, Vietnam. J Prev Med Public Health 41, 232-240.

31. Foster Z, Byron E, Reyes-Garcia V et al. (2005) Physical growth and nutritional status of Tsimane' Amerindian children of lowland Bolivia. Am J Phys Anthropol 126, 343-351.

32. Decaro JA, Decaro E \& Worthman CM (2010) Sex differences in child nutritional and immunological status 5-9 years post contact in fringe highland Papua New Guinea. Am J Hum Biol 22, 657-666.

33. Marshall LB (1985) Infant Care and Feeding in the South Pacific. New York: Gordon \& Breach.

34. Stinson S (1985) Sex differences in environmental sensitivity during growth and development. Am J Phys Anthropol 28, 123-147.
35. Simeon DT \& Grantham-McGregor S (1989) Effects of missing breakfast on the cognitive functions of school children of differing nutritional status. Am J Clin Nutr $\mathbf{4 9}$, 646-653.

36. Rampersaud GC, Pereira MA, Girard BL et al. (2005) Breakfast habits, nutritional status, body weight, and academic performance in children and adolescents. $\mathrm{J} \mathrm{Am}$ Diet Assoc 105, 743-760.

37. Black RE, Allen LH, Bhutta ZA et al. (2008) Maternal and child undernutrition: global and regional exposures and health consequences. Lancet 371, 243-260.

38. Burrows TL, Martin RJ \& Collins CE (2010) A systematic review of the validity of dietary assessment methods in children when compared with the method of doubly labeled water. J Am Diet Assoc 110, 1501-1510.

39. Roman-Vinas B, Ortiz-Andrellucchi A, Mendez $\mathrm{M}$ et al. (2010) Is the food frequency questionnaire suitable to assess micronutrient intake adequacy for infants, children and adolescents? Matern Child Nutr 6, Suppl. 2, 112-121.

40. Ng M, Fleming T, Robinson M et al. (2014) Global, regional, and national prevalence of overweight and obesity in children and adults during 1980-2013: a systematic analysis for the Global Burden of Disease Study 2013. Lancet 384, 766-781.

41. Division of Cancer Prevention and Control, Centers for Disease Control and Prevention (2010) American Samoa Comprehensive Cancer Control Factsheet Series 2010. http://medicaid.as.gov/wp-content/uploads/2015/10/AS_CCC_ Obesity_Fact-Sheet.pdf (accessed September 2016).

42. Paulino YC, Coleman P, Davison NH et al. (2008) Nutritional characteristics and body mass index of children in the Commonwealth of the Northern Mariana Islands. J Am Diet Assoc 108, 2100-2104.

43. Astrup A (2008) The role of calcium in energy balance and obesity: the search for mechanisms. Am J Clin Nutr $\mathbf{8 8}$, 873-874.

44. Pries AM, Huffman SL, Adhikary I et al. (2016) High consumption of commercial food products among children less than 24 months of age and product promotion in Kathmandu Valley, Nepal. Matern Child Nutr 12, Suppl. 2, 22-37.

45. Nauru Bureau of Statistics \& Macro International, Inc. (2009) Nauru 2007 Demographic and Health Survey. Noumea, New Caledonia: Secretariate of the Pacific Community.

46. Kemmer TM, Novotny R \& Ah Ping I (2008) Iron deficiency and anemia: disparity exists between children in American Samoa and children living within the US. Eur J Clin Nutr $\mathbf{6 2}$, 754-760.

47. Tzioumis E \& Adair LS (2014) Childhood dual burden of under- and overnutrition in low- and middle-income countries: a critical review. Food Nutr Bull 35, 230-243.

48. Schafer RB, Schafer E, Dunbar M et al. (1999) Marital food interaction and dietary behavior. Soc Sci Med 48, 787-796.

49. Combs-Orme T, Wilson EE, Cain DS et al. (2003) Contextbased parenting in infancy: background and conceptual issues. Child Adolesc Soc Work J 20, 437-472.

50. Bailey RL, West KP Jr \& Black RE (2015) The epidemiology of global micronutrient deficiencies. Ann Nutr Metab 66, Suppl. 2, 22-33.

51. Lopez A, Cacoub P, Macdougall IC et al. (2016) Iron deficiency anaemia. Lancet 387, 907-916.

52. Kassebaum NJ (2016) The global burden of anemia. Hematol Oncol Clin North Am 30, 247-308.

53. Sjölander K (2012) Prevalence of selected infectious disesaes in Samoan dogs. Master in Animal Science Thesis, Sveriges lantbruksuniversitet.

54. Eckhardt CL (2006) Micronutrient Malnutrition, Obesity, and Chronic Disease in Countries Undergoing the Nutrition Transition: Potential Links and Program/policy 
Implications. Washington, DC: International Food Policy Research Institute, Food Consumption and Nutrition Division.

55. Doak CM, Adair LS, Bentley M et al. (2005) The dual burden household and the nutrition transition paradox. Int $J$ Obes (Lond) 29, 129-136.

56. Garcia OP, Long KZ \& Rosado JL (2009) Impact of micronutrient deficiencies on obesity. Nutr Rev 67, 559-572.

57. Tzioumis E, Kay MC, Bentley ME et al. (2016) Prevalence and trends in the childhood dual burden of malnutrition in low- and middle-income countries, 1990-2012. Public Health Nutr 19, 1375-1388.

58. Hawkes C, Haddad L \& Udomkesmalee E (2015) The Global Nutrition Report 2015: what we need to do to advance progress in addressing malnutrition in all its forms. Public Health Nutr 18, 3067-3069.

59. Samoa Bureau of Statistics (2015) Samoa Demographic and Health Survey 2014. Apia, Samoa: Samoa Demographic and Health Survey, Government of Samoa. 